\title{
Time-resolved spectroscopy of the excited electronic state of reaction centers of $R$ hodopseudomonas viridis
}

\author{
P.O.J. Scherer ${ }^{\text {a,* }}$, S.F. Fischer ${ }^{\text {a }}$, C.R.D. Lancaster ${ }^{b}$, G. Fritzsch ${ }^{b}$, S. Schmidt ${ }^{\text {c }}$, T. Arlt ${ }^{\text {c }}$, \\ K. Dressler ${ }^{\mathrm{c}, 1}, \mathrm{~W}$. Zinth ${ }^{\mathrm{c}}$ \\ - Physik Department T 38, Technische Universität München, Boltzmannstraße, D-85748 Garching bei München, Germany \\ b Abteilung für molekulare Membranbiologie, Max-Planck-Institut für Biophysik, \\ Heinrich-Hoffmann-Straße 7, D-60528 Frankfurt, Germany \\ 'Institut fur medizinische Optik, Ludwig Maximilians Universität München, Barbarastraße 16, D-80797 München, Germany
}

Received 7 March 1994; in final form 29 March 1994

\begin{abstract}
The spectral properties of the excited electronic state of the reaction centers of Rhodopseudomonas (Rps.) viridis are studied by dichroic transient absorption spectroscopy with sub-picosecond time resolution. The theoretical analysis of the experimental results allows the assignment of the transient absorption from two dimer bands of the special pair and show its excitonic coupling to other pigments.
\end{abstract}

\section{Introduction}

The process of bacterial photosynthesis in reaction centers (RCs) starts at a strongly coupled pair of bacteriochlorophyll molecules, the primary donor $\mathrm{P}$ [1]. This so-called special pair is excited directly by light absorption or by energy transfer from antenna pigments. Exact knowledge of the properties of $P$ and of its excited electronic state $\mathrm{P}^{*}$ is essential for a better understanding of the first electron transfer reaction in bacterial photosynthesis. In this Letter, we present transient data from excite-and-probe experiments taken at early delay times and with different probing polarizations to characterize the initially excited electronic state $\mathbf{P}^{*}$ of the special pair. Analysis of the data based on quantum calculations of the ex-

\footnotetext{
* Corresponding author.

1 Physik Department E11, Technische Universität München, Boltzmannstraße, D-85748 Garching bei München, Germany.
}

cited states of $\mathbf{P}$ yields information on the excitonic couplings of the pigments in the reaction center and on the location of the upper electronic level $\mathrm{P}^{*}(+)$ of the special pair.

\section{Material and methods}

Experimental. The experiments were performed at room temperature on isolated reaction centers of $R p s$. viridis (wild type), prepared according to ref. [2]. The RCs were kept in a cell with $1 \mathrm{~mm}$ path length at concentrations yielding $O D=5-10 \mathrm{~cm}^{-1}$ at $960 \mathrm{~nm}$. Stirring ascertained the exchange of the sample between two laser shots. Less than $10 \%$ of the RCs in the irradiated volume were excited. The experimental apparatus for transient absorption spectroscopy has been described in detail earlier [3]. The exciteand-probe spectrometer is based on an amplified colliding pulse mode locked (CPM) dye laser. The pulses 
of this laser are frequency shifted by femtosecond continuum generation and, in the case of the excitation branch, reamplified a second time. Other salient features of the system are: repetition rate of $50 \mathrm{~Hz}$, wavelength of pump pulses at $\lambda=965 \mathrm{~mm}$ and spectral width of the probing pulses of $10-15 \mathrm{~nm}$. The instrumental response function, i.e. the cross correlation between excitation and probing pulses has a width of approximately $250 \mathrm{fs}$. Linear polarization of the pulses is better than $97 \%$. The polarization of the probing pulses relative to the exciting pulses is adjusted by tuning the polarization of the probe beam using an achromatic $\frac{1}{2} \lambda$ plate. Both beams cross in the sample at an angle of $8^{\circ}$ in a plane parallel to the exciting electric field vector. Transient absorbance changes were recorded at room temperature at different wavelength positions in the $500-1050 \mathrm{~nm}$ range for delay times of -1 to 10 ps. Each measurement was individually normalized on the excitation energy and optical density of the sample. The absorbance change due to the population of the excited electronic level of $\mathrm{P}^{*}$ was deduced by taking the measured absorbance change at a delay time $t_{D}=250 \mathrm{fs}$,

$$
\begin{aligned}
& \Delta A_{\mathrm{p} *}(\lambda)=A_{\mathrm{p} *}(\lambda)-A_{\text {ground }}(\lambda) \\
& \quad \approx A\left(t_{\mathrm{D}}=250 \mathrm{fs}, \lambda\right)-A(-\infty, \lambda) .
\end{aligned}
$$

\section{Results}

\subsection{Ground state spectrum}

The absorption spectra of the reaction centers of Rps. viridis is presented in Fig. 1a (filled circles). The broad absorption band at $960 \mathrm{~nm}$ is due to the lowenergy electronic transition $\mathrm{P} \rightarrow \mathrm{P}^{*}(-)$ of the special pair. The $Q_{y}$ transitions of the other pigments occur at shorter wavelengths at $830 \mathrm{~nm}$ (accessory-bacteriochlorophylls $B_{A, B}$ ) and around $800 \mathrm{~nm}$ (bacteriopheophytins $\mathrm{H}_{\mathrm{A}, \mathrm{B}}$ ). The corresponding $\mathrm{Q}_{x}$ transitions occur at $605 \mathrm{~nm}\left(\mathrm{P}\right.$ and $\left.\mathrm{B}_{\mathrm{A}, \mathrm{B}}\right)$ and $540 \mathrm{~nm}$ $\left(\mathrm{H}_{\mathrm{A}, \mathrm{B}}\right)$.

\subsection{Instantaneous absorbance changes}

Transient absorbance changes $\Delta A_{\mathrm{P} *}$ are displayed in Fig. $2 b$ for the spectral range $500-1050 \mathrm{~nm}$ for a

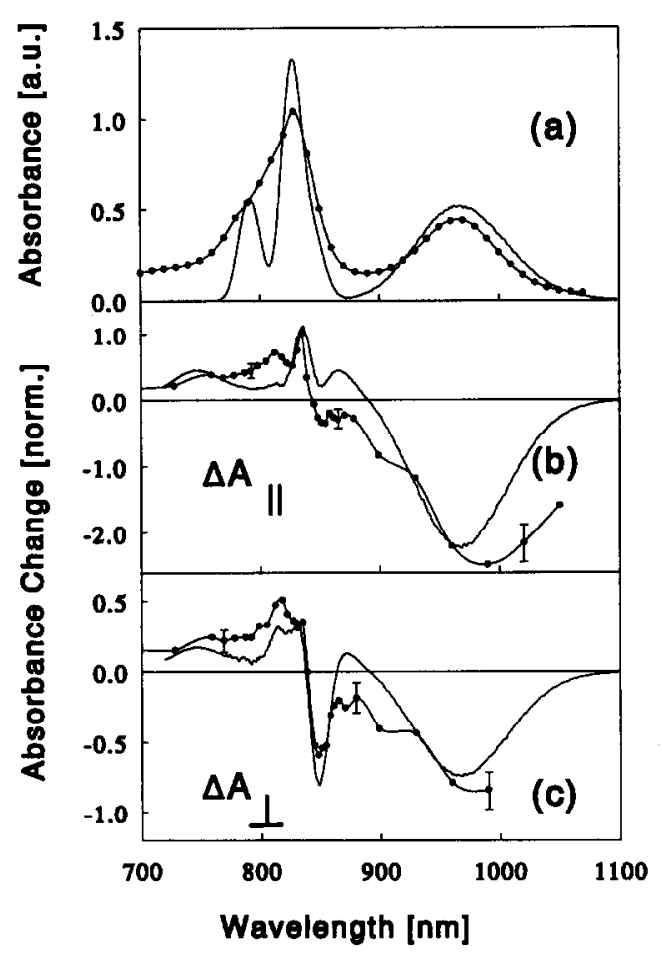

Fig. 1. Experimental data (closed circles) and simulations (solid lines without circles) of reaction centers of Rps. viridis. stationary absorbance (a) and the induced absorbance changes ( (b), (c) ) at a delay time $t_{\mathrm{D}}=250 \mathrm{fs}$, yielding the difference spectrum between the excited electronic state of the special pair $\mathrm{P}^{*}$ and the initial ground state. Parallel (b) as well as perpendicular (c) polarizations of the probing pulses are used.

parallel polarization of exciting and probing pulses (filled circles). In the long-wavelength side of the special pair absorption band a strong absorbance decrease occurs, which is due to the stimulated emission from the excited electronic state of the special pair. Towards shorter wavelengths $(\lambda \approx 950 \mathrm{~nm})$ one observes the bleaching of the special pair ground state absorption. In the short-wavelength wing of this band around $860 \mathrm{~nm}$ there is a striking modulation of the absorbance changes: the minimum of absorption at $855 \mathrm{~nm}$ is directly neighboured by a narrow (width $\approx 15 \mathrm{~nm}$ ) positive absorption peak at $830 \mathrm{~nm}$, while a broader peak occurs at $810 \mathrm{~nm}$. Towards shorter wavelengths, down to $500 \mathrm{~nm}$, there is a broad absorbance increase with a weak dip in the $Q_{x}$ band of the bacteriochlorophylls around $610 \mathrm{~nm}$. Of special interest is the region between 790 and $870 \mathrm{~nm}$ where the bacteriopheophytins and the accessory bacteriochlorophylls absorb and where the upper ex- 


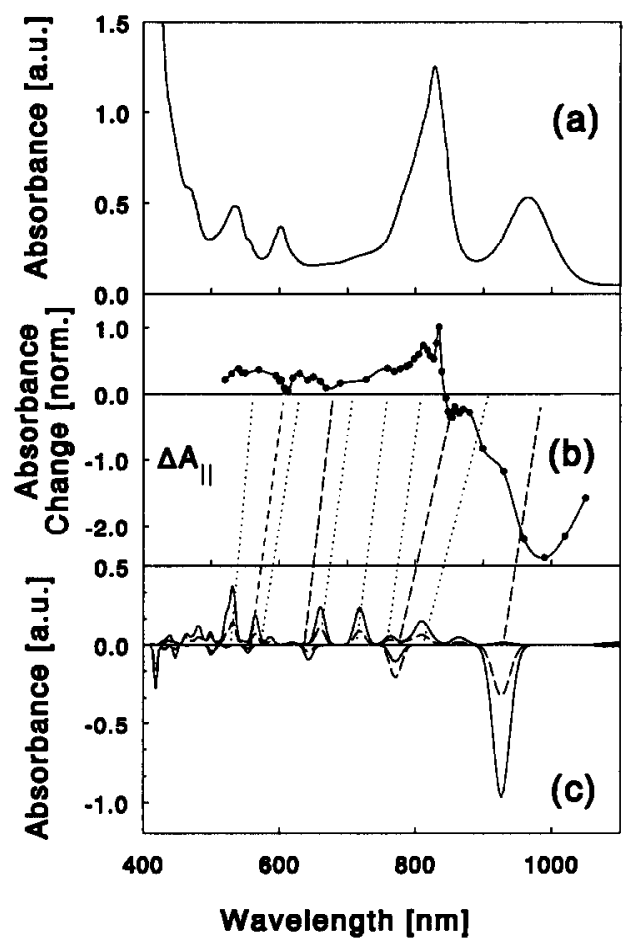

Fig. 2. Experimental ground state absorption (a) together with the measured transient absorption changes ( $b$, parallel polarization of exciting and probing pulses) and the results of the quantum calculations for the ground state spectrum of $P$ (negative going in $c$ ) as well as the excited state spectrum of $P^{*}$ (positive part of c). Solid curves represent parallel polarizations, broken curves perpendicular polarizations. The broken and dotted lines correlate absorption bands in the theoretical simulation with the corresponding features in the experimental spectrum for the ground state $P$ and the excited state $P^{*}$, respectively.

citonic transition of the special pair should be located. Additional information for this spectral range can be obtained by comparing experiments with perpendicular and parallel polarization: Figs. $1 \mathrm{~b}$ and $1 \mathrm{c}$ show the results of the dichroic measurements between 700 and $1050 \mathrm{~nm}$ on an enlarged scale. The negative band at $855 \mathrm{~nm}$, which is not very pronounced for parallel polarization (Fig. 1b), stands out in perpendicular polarization (Fig. 1c). The opposite is found for the narrow positive band at $830 \mathrm{~nm}$ which is clearly seen in the parallel polarization experiment. The broad increased absorption at still shorter wavelengths has only a weak dichroism. We obtain here an average dichroic ratio of $\Delta A_{\|}$/ $\Delta A_{\perp}=1.4 \pm 0.3$. Of interest is the polarization dependency also in the region of the dimer $Q_{y}$ band
(900-1000 nm), where we obtain a value of $\Delta A_{\|}$' $\Delta A_{\perp}=2.8 \pm 0.3$, close to three. Before we present detailed theoretical simulations of these data (solid lines without dots) we want to discuss the experimental results qualitatively.

\subsection{Analysis}

To analyze the data presented here, we must keep in mind that the recorded spectra reflect the absorption changes immediately after optical excitation at a delay time of $\approx 250 \mathrm{fs}$. These properties are averaged over the pulse duration, i.e. the experimental width of the instrumental response function. At $t_{\mathrm{D}}=250 \mathrm{fs}$ the reaction centers are predominantly in the state $P^{*}$. As a consequence the qualitative interpretation of the special features is based on the following considerations: positive bands appear if there is a new absorption created upon excitation of the special pair. Such a band can be due to the excited state absorption of $\mathrm{P}^{*}$ or to the reappearance of a ground state band of another chromophore in the reaction center, whose spectral properties were modified by changes in the couplings between the excited states. Negative bands indicate a disappearance of the absorption present in the reaction center's ground state or a stimulated emission from the special pair.

The dichroic ratio close to 3 in the spectral range of the stimulated emission and in the dimer $Q_{y}$ band around $960 \mathrm{~nm}$ indicates that the detected transition dipole moments are parallel to the excited transition moment of the special pair ground state. Apparently, no reorientation of the transition dipole moment of $P$ takes place upon optical excitation within $250 \mathrm{fs}$.

For the absorbance at $855 \mathrm{~nm}$ we obtain a dichroic ratio of $\Delta A_{\sharp} / \Delta A_{\perp} \approx 0.6$. This value indicates that the related transition direction is approximately perpendicular to the absorption dipole moment of the excited $Q_{y}$ transition. This finding is well understood if this band is due to the upper electronic transition $\mathrm{Q}_{y^{+}}(\mathrm{P})$ of the special pair. Further experimental evidence for this assignment of the observed $855 \mathrm{~nm}$ band has been given by steady-state dichroic data [4] and hole-burning experiments [5].

In that case the adjacent strong positive absorption band at $830 \mathrm{~nm}$ and their interactions with the upper dimer band prevent a high precision determination of the exact spectral position of this $P^{*}(+)$ band. The 
position of the peak $830 \mathrm{~nm}$ coincides well with the maximum of the $Q_{y}$ absorption of the monomeric bacteriochlorophyll molecules. Therefore, we assign this band to an increase in the $\mathrm{BChl}$ absorption upon formation of the excited electronic state $\mathrm{P}^{*}$. The dichroism of this band $\Delta A_{1} / \Delta A_{\perp} \approx 2.4$ yields an angle of approximately $22^{\circ}$. This is in qualitative agreement with the value expected for the bacteriochlorophyll monomer from the X-ray structure of the RC. The assignment of this band is consistent with former low-temperature results on $\mathrm{RC}$ of $R b$. capsulates and $R b$. sphaeroides presented in refs. $[4,6]$.

\section{Theoretical analysis}

The aim of our theoretical analysis is twofold. First, we want to present quantum calculations on the dimer, especially the excited state spectrum. Secondly, we want to use this information to simulate the transient spectra including the other pigments. This will be done on the grounds of an extended exciton model [7] including line-shape effects.

\subsection{Quantum analysis}

We use a semi-empirical program which is based on the INDO-S Hamiltonian [8]. Such an approach has been shown to give a quite reasonable description of the dimer absorption spectrum and was also successfully applied to the whole hexamer of the reaction center (4 BChl's, 2 BPhe's plus some protein residues) $[7,9]$. For calculations on excited state absorption spectra it is essential to include multiple substituted configurations in the $\mathrm{CI}$ basis. Here, we consider single, double and triple excitations and make use of spin adapted combinations to reduce the number of basis states. The most important 300 configurations are treated exactly and a further 10000 perturbationally [9]. The parameterization is essentially that of Zerner [8].

The resulting spectrum of the dimer ground state absorption shows the strong $Q_{y}$ transition $\left(\mathrm{P} \rightarrow \mathrm{P}^{*}(-)\right)$ at $\approx 950 \mathrm{~nm}$ and the so-called upper dimer band $\left(\mathrm{P} \rightarrow \mathrm{P}^{*}(+)\right)$ at $\approx 780 \mathrm{~nm}$ which has less intensity and is polarized almost perpendicular to the lower dimer band. The lowest intradimer charge transfer states appear at $\approx 500 \mathrm{~nm}$. The spectrum is shown in Fig. 2c (lower part). The calculated line spectrum was dressed with a Gaussian line profile with a half-width of $100 \mathrm{~cm}^{-1}$. The solid line gives the parallel polarization, the dashed line presents the perpendicular polarization.

The calculated spectrum of $P^{*}$ (upper part of Fig. 2c) shows a broad distribution of transitions which starts around the upper dimer band and extends to higher energies. Some distinct transitions appear between 600 and $850 \mathrm{~nm}$ which are polarized nearly parallel to the lower dimer band $\left(\leqslant 30^{\circ}\right)$.

\subsection{Simulations}

The ground state absorption spectrum is simulated within an excitonic model which treats the dimer as a supermolecule [7]. The excitonic couplings $V_{m, n}$ to and among the other pigments are calculated within the transition dipole approximation using transition dipole vectors from a quantum chemical calculation and a shielding factor of 0.15 that accounts for the overestimation of transition dipoles by the semi-empirical program and the dielectric shielding in the protein environment. Comparing with quantum calculations the dimer transitions have to be shifted 600 $\mathrm{cm}^{-1}$ to lower energies and the energy splitting between the two dimer transitions has to be reduced by $750 \mathrm{~cm}^{-1}$. This is necessary as the semi-empirical parameters are optimized for gas phase calculations without multiply substituted configurations.

Diagonalization of the interaction matrix provides the positions and intensities of the exciton transitions. For comparison with experimental data the line profiles have to be simulated. Usually the calculated stick spectra are simply dressed by Gaussian profiles. This procedure, however, becomes questionable if overlapping transitions with different widths are coupled. The eigenstates are strongly mixed and it becomes even quite unclear, which widths should be applied to the mixed states. Here, a more careful simulation of the line profile is necessary which explicitly takes into account the inhomogeneous broadening of the excitonic transitions [10]. This is achieved by adding a diagonal disorder contribution $\delta_{m}$ to the energies $E_{m}$ and averaging over independent Gaussian distributions (uncorrelated disorder is assumed), i.e. the energies of the bare states rather than 
the exciton states are broadened. The model Hamiltonian,

$H=\sum|m\rangle\left\langle m\left|\left(E_{m}+\delta_{m}\right)+\sum\right| m\right\rangle\langle n| V_{m, n}$

(see Table 1), is diagonalized for $10^{5}$ different choices of the diagonal shifts $\delta_{m}$ which are obtained from a Gaussian random number generator [11]. Such a treatment is necessary for spectra consisting of overlapping bands, especially for the $\mathbf{P}^{*}$ contributions which are expected to have a larger width than the monomer band in the same wavelength region. For separated bands or weak excitonic coupling the resulting spectrum is just the sum of Gaussian functions at the exciton transitions. This holds especially for the ground state spectrum of the reaction center.

The excited state $\mathrm{P}^{*}$ spectrum is calculated in a similar way but with the dimer states $P^{*}( \pm)$ replaced by several excitations of the dimer (Table 2 ). From the quantum results seven excited states in the wavelength region between 600 and $1000 \mathrm{~nm}$ are taken into account (denoted as $P_{1}^{-*}$ to $P_{7}^{* *}$ ).

This whole set of transitions was red-shifted by 700 $\mathrm{cm}^{-1}$ to achieve agreement with the experiments. These $\mathrm{P}^{*}$ excitations are coupled strongly to the monomer band which gains additional intensity. Comparing with the experimental spectra we conclude that the excitonic coupling between dimer and accessory monomers should not exceed some $70 \mathrm{~cm}^{-1}$, which is consistent with our shielding factor.

In the simulation the linewidth was reduced as compared to the experimental ground state absorption to account for the selectivity of the excitation process similar to ADMR spectra. We further used a smaller width for the perpendicular polarized peak of $\mathrm{P}_{3}^{* *}$ as compared to the parallel polarized peaks. This is motivated by the fact that only the parallel polarized components of the special pair transitions can couple well to the internal charge-transfer states. Experimentally this is seen in the larger width and stronger electrochromicity of the lower dimer band as compared to the upper.

\section{Interpretation}

In Figs. $2 \mathrm{~b}$ and $2 \mathrm{c}$ we tried to correlate the quantum calculations for the dimer with special features of the transient absorption changes. The dashed lines refer to the ground state spectrum, which is bleached after excitation and, therefore, is shown as the negative part of Fig. 2c. There is a good correlation with regard to energy position, intensity and polarization if the calculated spectrum is slightly shifted to lower energies. This correction is necessary as the semi-empirical method is parameterized for calculations in the gas phase and does not include environmental shifts.

In particular, the lower and the upper dimer bands are well represented for both the parallel and perpendicular polarization directions as can be seen also in the simulation of Figs. $1 b$ and $1 c$. The quantum calculations also give an intensity corresponding to the experimental dip around $600 \mathrm{~nm}$ which should represent the $Q_{x}$ transition of the dimer. There is, however, strong mixing with internal charge transfer states showing up around $500 \mathrm{~nm}$. The spectrum of $\mathrm{P}^{*}$ (positive part of Fig. 2c) is correlated with experiment in Fig. $2 b$ via dotted lines. Here, the assignment

Table 1

Interaction matrix elements $E_{m}$ and $V_{m, n}\left(\mathrm{~cm}^{-1}\right)$, intensities $\mu^{2}\left(\mathrm{D}^{2}\right)$ and Gaussian widths $\Delta\left(\mathrm{cm}^{-1}\right)$ for the ground state absorption spectrum

\begin{tabular}{|c|c|c|c|c|c|c|}
\hline & $P^{*}(-)$ & $P^{*}(+)$ & $\mathbf{B}_{\mathbf{M}}^{*}$ & $\mathbf{B}_{\mathbf{L}}^{*}$ & $\mathbf{H}_{\mathbf{M}}^{*}$ & $\mathbf{H}_{\mathbf{L}}$ \\
\hline $\mathrm{P}^{*}(-)$ & 10336 & 0 & 62 & -62 & 9 & 9 \\
\hline$P^{*}(+)$ & & 11800 & 10 & 10 & 0 & 0 \\
\hline $\mathbf{B}_{\mathbf{M}}^{*}$ & & & 12030 & 0 & -50 & 0 \\
\hline $\mathrm{B}_{\mathbf{L}}^{\mathbf{E}}$ & & & & 12130 & 0 & -50 \\
\hline $\mathbf{H}_{\mathbf{M}}^{*}$ & & & & & 12700 & 0 \\
\hline $\mathrm{H}_{\mathbf{L}}^{*}$ & & & & & & 12550 \\
\hline$\mu^{2}$ & 153 & 38 & 85 & 85 & 60 & 60 \\
\hline$\Delta$ & 560 & 170 & 170 & 170 & 170 & 170 \\
\hline
\end{tabular}


Table 2

Diagonal energies $E_{m}\left(\mathrm{~cm}^{-1}\right)$ of the $\mathrm{P}$ excitations, intensities $\mu^{2}\left(D^{2}\right)$, angle $\alpha$ of the transition moment, Gaussian half-widths $\Delta\left(\mathrm{cm}^{-1}\right)$ and excitonic coupling to the accessory monomers

\begin{tabular}{lrrlll}
\hline$E$ & $\mu^{2}$ & \multicolumn{1}{c}{$\alpha$} & $\Delta$ & $V\left(\mathrm{P}^{* *}, \mathrm{~B}_{\mathrm{M}}^{*}\right)$ & $V\left(\mathrm{P}^{* *}, \mathrm{~B}_{\mathrm{L}}^{*}\right)$ \\
\hline 11650 & 9 & $8^{\circ}$ & 500 & 15 & -15 \\
11827 & 26 & $17^{\circ}$ & 500 & 25 & -25 \\
12260 & 8 & $81^{\circ}$ & 170 & 6 & 6 \\
12560 & 11 & $29^{\circ}$ & 500 & 17 & -17 \\
13366 & 30 & $8^{\circ}$ & 500 & 28 & -28 \\
13543 & 5 & $41^{\circ}$ & 500 & 11 & -11 \\
14700 & 35 & $16^{\circ}$ & 500 & 30 & -30 \\
\hline
\end{tabular}

is not so apparent since the spectra are broad. Nonetheless, we find that not only the predicted overall intensity increase is consistent with the measurements but also the intensity distribution in the 540 $\mathrm{nm}$ range and the $800 \mathrm{~nm}$ range are well represented. There is also one state with a perpendicular component on the short wavelength side of the upper dimer band. One might interpret this as the upper dimer band of the excited dimer. However, the calculated intensity $\left(8 D^{2}\right)$ is too small to explain the experimentally observed perpendicular absorption. Agreement with the experimental spectrum could be only achieved if the intensity of this state would be increased by a factor of 2.5 . This is of special interest, since it may tell us something about structural changes of the excited state. If there would be a symmetry breaking relaxation such that the excited state transitions acquire a stronger perpendicular component, this feature could be well understood. At this point we would like to remind that earlier calculations on the dimer [7] showed too little dipolar changes due to excitation as reflected in a too small Stark amplitude. This shortcoming of the calculations may indeed have the same common origin, that is the neglect of distortions of the excited dimer.

Another interesting result of the calculations is the rather sharp structure in the monomer range and the intensity increase of the monomer band. The intensity increase has two origins. In the ground state spectrum the intensity is shifted to the dimer. This inten- sity loss disappears when the dimer is excited. Furthermore, the excited states of $P^{*}$ transfer the intensity into the monomer band, since most of this intensity resides in the shorter wavelength region. There is also a small shift of the monomer band to longer wavelengths. We found it essential to represent the broad transient absorption band around $800 \mathrm{~nm}$ by a distribution of many states. Only in this way the relative small width of the monomer band in Figs. 1b, $1 c$ and $2 b$ could be simulated.

In summary, the presented transient spectra of the excited electronic state of the special pair can be well interpreted by semi-empirical quantum calculations, providing new information on the structure of the excited state of the special pair.

\section{References}

[1] J. Deisenhofer and J.R. Norris, eds., in: The photosynthetic reaction center, Vols. I+II (Academic Press, New York, 1993).

[2] K. Dressler, E. Umlauf, S. Schmidt, P. Hamm, W. Zinth, S. Buchanan and H. Michel, Chem. Phys. Letters 183 (1991) 270.

[3] S. Schmidt, T. Arlt, P. Hamm, C. Lauterwasser, U. Finkele, G. Drews and W. Zinth, Biochim. Biophys. Acta 1144 (1993) 385.

[4] G. Paillotin, A. Vermeglio and J. Breton, Biochim. Biophys. Acta 545 (1979) 249.

[5] D. Tang, S.G. Johnson, R. Jankowiak, J.M. Hayes, G.J. Small and D.M. Tiede, in: Perspectives in photosynthesis, eds. J. Jortner and B. Pullman (Kluwer, Dordrecht, 1990) p. 99.

[6] M.H. Vos, F. Rappaport, J.-C. Lambry, J. Breton and J.-L. Martin, in: NATO ASI Series A237. The photosynthetic bacterial reaction center II, eds. J. Breton and A. Verméglio (Plenum Press, New York, 1992) p. 237.

[7] P.O.J. Scherer and S.F. Fischer, in: Chlorophylls, ed. H. Scheer (CRC Press, Boca Raton, 1991) p. 1079.

[8] W.D. Edwards, J.D. Head and M.C. Zerner, J. Am. Chem. Soc. 104 (1982) 5833.

[9] P.O.J. Scherer, J. Luminescence 53 (1992) 133.

[10] H. Fidder, J. Knoester and D.A. Wiersma, J. Chem. Phys. 95 (1991) 7880.

[11] W.H. Press, B.P. Flannery, S.A. Teukolsky and W.T. Vetterling, in: Numerical recipes (Cambridge Univ. Press, Cambridge, 1986) pp. $200 f f$. 